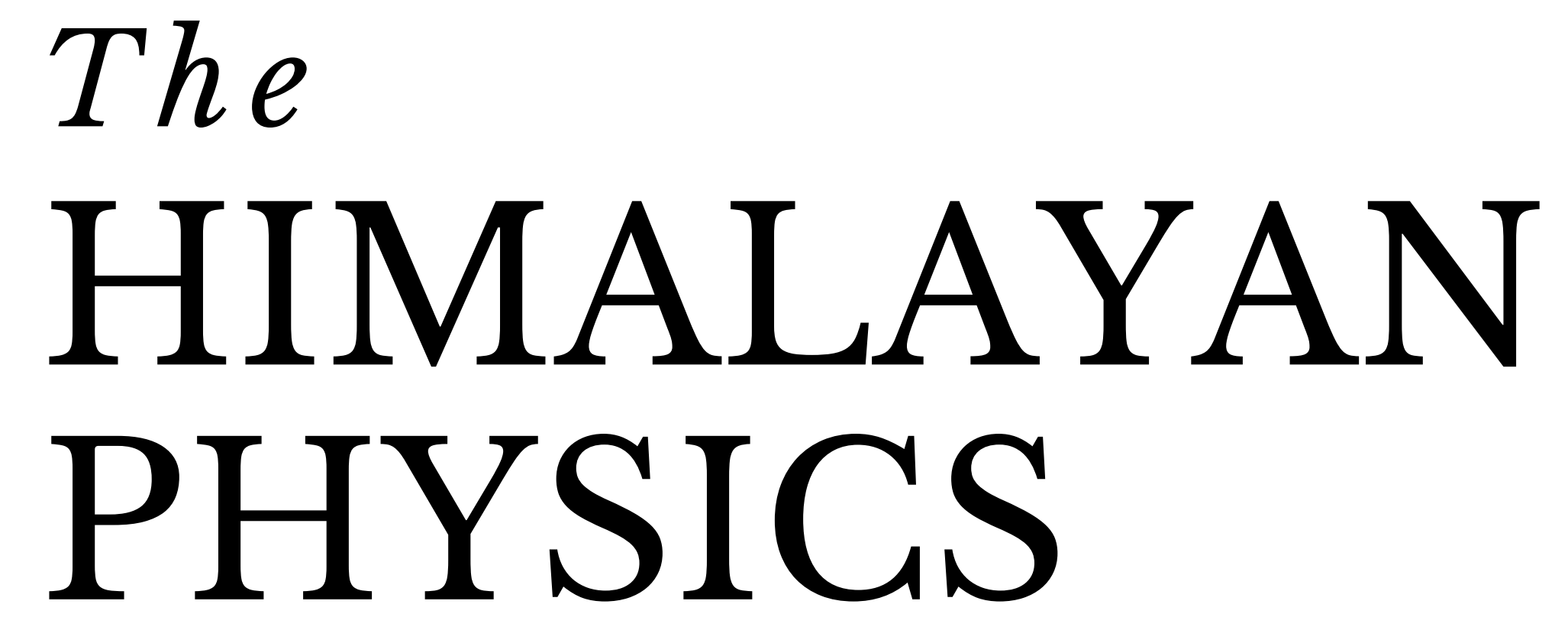

A peer-reviewed Journal of Physics

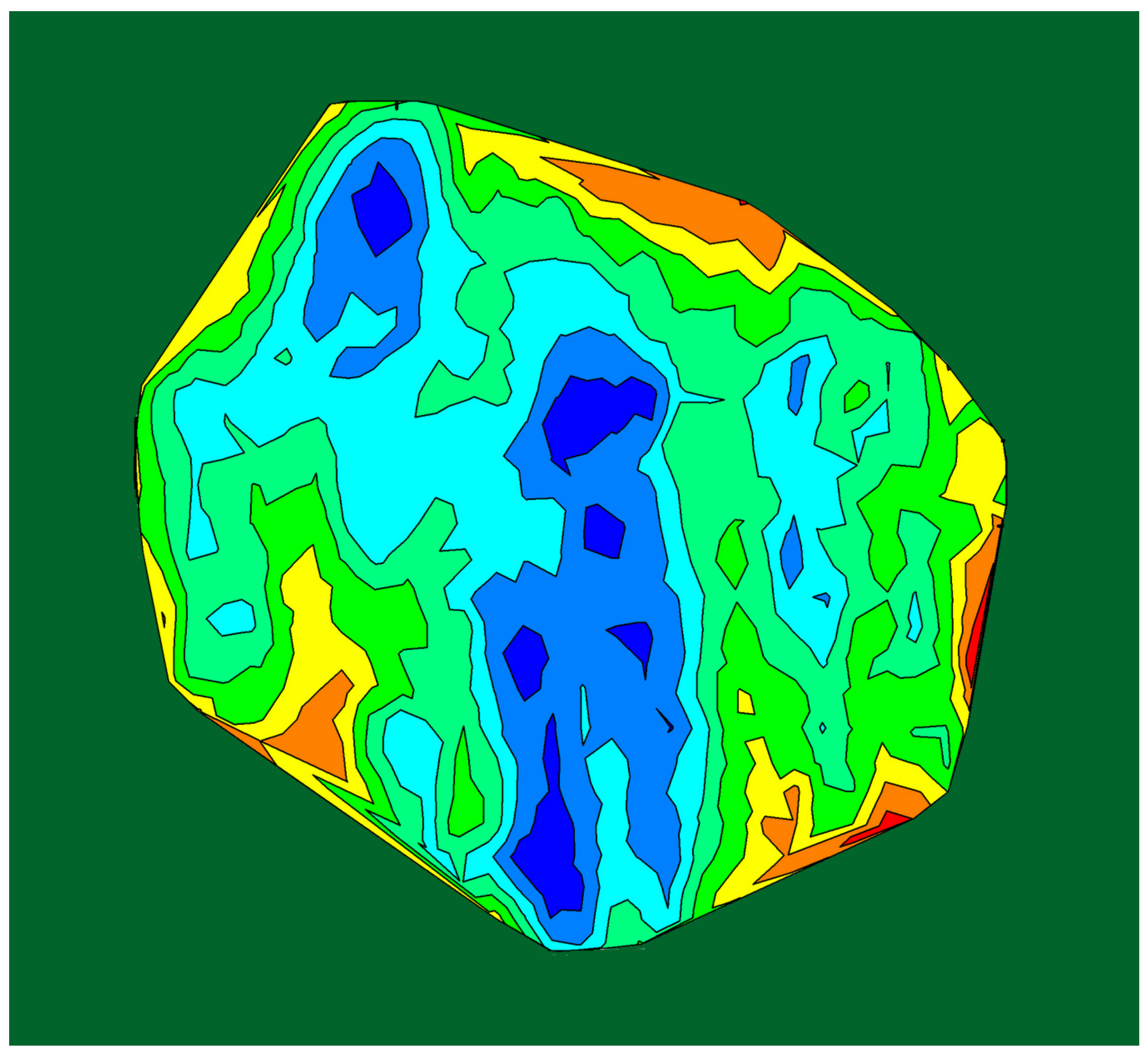

Department of Physics, Prithvi Narayan Campus, Pokhara Nepal Physical Society, Western Chapter, Pokhara 


\section{Publisher}

Department of Physics, Prithvinarayan Campus, Pokhara Nepal Physical Society, Western Chapter, Pokhara

\section{The Himalayan Physics}

Volume 9, December 2020

ISSN 2542-2545

The Himalayan Physics (HimPhys) is an open access peer-reviewed journal that publishes quality articles which make innovative contributions in all areas of Physics. HimPhys is published annually by Nepal Physical Society (Western Regional Chapter), and Department of Physics, Prithvi Narayan Campus, Pokhara. The goal of this journal is to bring together researchers and practitioners from academia in Nepal and abroad to focus on advanced techniques and explore new avenues in all areas of physical sciences and establishing new collaborations with physics community in Nepal.

\section{Chief Editor}

Kapil Adhikari

\section{Associate Editor \\ Aabiskar Bhusal}

(C)2020, Publishers. All rights reserved.

This publication is in copyright. Subject to statutory exception and to the provisions of relevant collective licensing agreements, no reproduction of any part may take place without written permission of the publishers.

Cover: Contour map of dust mass. (C) Mijas Tiwari. Printed from article in the current issue, with permission. 


\section{The HIMALAYAN PHYSICS}

A peer-reviewed Journal of Physics

\section{Chief Editor}

Kapil Adhikari

Associate Editor

Aabiskar Bhusal

\section{Publisher}

Department of Physics, Prithvi Narayan Campus, Pokhara Nepal Physical Society, Western Chapter, Pokhara 


\section{Nepal Physical Society Western Regional Chapter Pokhara, Nepal}

\section{President}

Min Raj Lamsal

Immediate Past President

Jeevan Regmi

Vice-President

Sundar Prasad Dhakal

Secretary

Ravi Karki

Treasurer

Dipak Adhikari

Joint Secretary

Sujan Lamsal

Editorial Member

Kapil Adhikari

Members

Amrit Dhakal

Laxman Thapa

Laxman Timilsina

Narayan Prasad Bhandari

Pradeep Subedi

Advisory Board

Prof. Dr. Pradip K. Bhattarai

Pabitra Mani Poudyal

Surya Bahadur G.C.

Parashu Ram Poudel

Prof. Dr. Shovakanta Lamichhane

Kul Prasad Dahal

Dr. Krishna Raj Adhikari

Ram Sajile Verma 


\section{Himalayan Physics Vol-9 (2020)}

\section{TABLE OF CONTENTS}

Metal Organic Frameworks(MOFs) as efficient carrier for targeted nanodrug delivery

R. Karki, D. Adhikari, K. Adhikari, N. Pantha

A Density Functional Theory Study on Paracetamol-Oxalic Acid Co-Crystal

P. Paudel, K.R. Adhikari, K. Adhikari

First-principles study of $\mathrm{C}$ cites vacancy defects in water adsorbed graphene

H.K. Neupane, N.P. Adhikari

Diusion of fructose in water: a molecular dynamics study

S. Bhusal, N. Pantha

Study of aecting factors of meteorological parameters on solar radiation on Pokhara P.M. Shrestha, J. Regmi, U. Joshi, K.N. Poudyal, N.P. Chapagain, I.B. Karki

Variation of mean value of velocity of ion with dierent obliqueness of magnetized plasma sheath

B.R. Adhikari, H.P. Lamichhane, R. Khanal

Study of dust properties of two far infrared cavities nearby asymptotic giant branch stars under infrared astronomical satellite maps

M. Tiwari, S.P. Gautam, A. Silwal, S. Subedi, A. Paudel, A. K. Jha

An experimental study on irradiated interface of silicon

M.R. Lamsal

Calculation of energy loss of proton beam on thyroid tumor

K. Giri, B. Paudel, B.R. Gautam

Study of noise level status at dierent rice mills in Surkhet Valley, Nepal

D.R. Paudel, H.N. Baral

Elliptically polarized laser assisted elastic electron-hydrogen atom collision and differential scattering cross-section

K. Yadav, S.P. Gupta, J.J. Nakarmi

Geodynamics of Gorkha earthquake (Mw 7.9) and its aftershocks

R.K. Tiwari and H. Paudyal 


\title{
Study of Dust Properties of two Far Infrared Cavities nearby Asymptotic Giant Branch stars under Infrared Astronomical Satellite Maps
}

Research Article

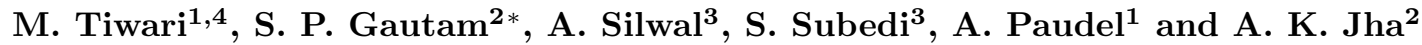 \\ 1 Department of Physics and Astrophysics, University of Delhi, Delhi-11007, India \\ 2 Central Department of Physics, Tribhuvan University, Kritipur, Nepal \\ 3 Patan Multiple Campus, Tribhuvan University, Lalitpur, Nepal \\ 4 Physics Research Initiatives (PRI), Pokhara, Nepal
}

\begin{abstract}
The physical properties such as dust color temperature, dust mass, visual extinction, and Planck function with their distribution in the core region of two far-infrared cavities, namely FIC16-37 (size $\sim 4.79 \mathrm{pc} \times 3.06 \mathrm{pc}$ ) located at R.A. (J2000): $16^{h} 33^{m} 57.25^{s} \&$ Dec. (J2000): $-37^{d} 47^{m} 04.3^{s}$, and FIC12-58 (size $\sim 22.54 \mathrm{pc} \times$ $14.84 \mathrm{pc}$ ) located at R.A. (J2000): $12^{h} 52^{m} 50.08^{s}$ \& Dec. (J2000): $-58^{d} 08^{m} 55.02^{s}$, found within a galactic plane $-10^{\circ}$ to $+10^{\circ}$ nearby Asymptotic Giant Branch (AGB) stars namely AGB15-38 (R.A. (J2000): $15^{h} 37^{m}$ $40.74^{s}$ \& Dec. (J2000): $-38^{d} 20^{m} 24.6^{s}$ ), and AGB12-57 (R.A (J2000): $12^{h} 56^{m} 38.50^{s}$ \& Dec. (J2000): $\left.-57^{d} 54^{m} 34.70^{s}\right)$, respectively were studied using Infrared Astronomical Satellite (IRAS) survey. The dust color temperature was found to lie in the range of $23.95 \pm 0.25 \mathrm{~K}$ to $23.44 \pm 0.27 \mathrm{~K}$ with an offset about $0.5 \mathrm{~K}$ for FIC16-37, and $24.88 \pm 0.27 \mathrm{~K}$ to $23.63 \pm 0.98 \mathrm{~K}$ with an offset about $1 \mathrm{~K}$ for FIC12-58. The low offset in the dust color temperature indicated the symmetric distribution of density and temperature. The total mass of the cavities FIC16-37 and FIC12-58 were found to be $0.053 \mathrm{M}_{\odot}$ and $0.78 \mathrm{M}_{\odot}$, respectively. The contour plots of mass distribution of both of the cavities was found to follow the cosmological principle, suggesting the homogeneous and isotropic distribution of dust masses. The plot between temperature and visual extinction showed a negative correlation, suggesting that higher temperature has lower visual extinction and vice-versa. The distribution of Planck function along major and minor diameters of both of the cavities was found to be non-uniform, indicating oscillation of dust particles to get dynamical equilibrium. It further suggested the role of pressure-driven events nearby both cavities and suggested that dust particles are not in thermal equilibrium along the diameters.

Keywords: Dust color temperature $\bullet$ dust mass $\bullet$ cavity $\bullet$ AGB star $\bullet$ ISM $\bullet$ IRAS
\end{abstract}

\section{Introduction}

The last evolutionary phases of a low and intermediate-mass of stars $\left(\mathrm{M}<10 \mathrm{M}_{\odot}\right)$ is called Asymptotic Giant Branch (AGB) . Their surroundings behave like the natural laboratory in which one can study their effects on Interstellar Medium (ISM) [1]. As the star runs out of hydrogen fuel at its core, the core starts to contract,

* Corresponding Author: astrosujan@gmail.com 
increasing its temperature. This causes the outer layer of the star to expand and cool, which results in their shift to a red giant, upper-right corner of HR diagram [2]. At about the temperature of $3 \times 10^{8} \mathrm{~K}$, helium burning begins in the core of star which halts further cooling and increases their luminosity [3]. This phase is well described by the horizontal branch in the HR diagram [2]. This phenomenon is observed for stars more massive than about 2 $\mathrm{M}_{\odot}[4]$. As the helium fusion completes in the core, the star moves towards the AGB region, right and upward region in the HR diagram, which then cools and expands their size as a result of an increase in luminosity [4].

One can describe the phenomena in AGB using a hydrogen-burning shell which lies below the convective envelope and a helium burning shell that lies above the electron-degenerate core of carbon and oxygen, or for massive AGB stars, there is a core of oxygen, neon, and magnesium [5]. The primary source of energy during the early AGB (E-AGB) phase is the helium fusion in a shell around the core of carbon and oxygen, and during this phase, the star becomes giant and switches to red giant again [6]. As the helium source runs out at the core, the thermally pulsating AGB (TP-AGB) phase starts and in this phase, the energy in AGB is from the fusion of hydrogen in a thin shell which results in a thin layer of inner helium shell and prevents their fusion [6]. Nevertheless, over the long period (10,000-100000 years), hydrogen-burning builds the helium which results in helium shell flash due to ignition of helium shell, that causes an increase in luminosity of the shell about thousands of times the total luminosity of stars, moreover, this event causes the expansion of the star and eventually cools resulting a convection zone between two shells [6]. The increase in the visible brightness of stars due to helium flash is common in AGB stars [7]. Material from the core region of the star is dredged-up into outer layers during thermal pulses, changing surface composition [8, 9]. This causes loss of significant mass of almost all (90-98\%) stars on the AGB in the form of massive winds [10].

Suh \& Kwon $[11,12]$ developed a complete catalogue of AGB stars in Infrared Astronomical Satellite (IRAS) Point Source Catalogue (PSC). Wood et al. [13] studied the images of nearby 100 dark molecular clouds at $60 \mu m$ and $100 \mu \mathrm{m}$ wavelengths using the all-sky database of IRAS. They proposed an empirical formula connecting optical depths, visual extinction of dust. Odenwald \& Rickard [14] and Odenwald [15] studied far-IR properties of 15 high galactic latitude clouds in $100 \mu \mathrm{m}$ IRAS maps. Gautam \& Aryal [1] detected an infrared nebula shaped by pulsar PSR B0823+26. Weinberger \& Armsdorfer [16] studied jet-like structure $\left(\sim 9^{\circ}\right)$ in a far-infrared region and concluded that this structure was formed because of the interaction of AGB wind with the ambient matter. Gautam et al. [17] studied dust color temperature and Planck function distribution of far infrared planetary nebula using AKARI survey, resulting high offset in dust color temperature and sinusoidal distribution of Planck function along major diameter of the structure. Aryal et al. [18] found two giant bipolar dust emission structures centred on PN NGC 1514 at FIR wavelengths. Aryal et al. [19] investigated the quadrupole cavity with PN NGC 2899 at its centre. Jha et al. [20] studied the dust color temperature and dust mass distribution in four lowlatitude $\left(|l|<20^{\circ}\right)$ far-infrared loops. Their work suggested the high-pressure events, and supernova explosion for the formation of these loops. Moreover, Jha \& Aryal [21] studied dust color temperature of two far-infrared cavities using AKARI and IRIS maps. 
In this work, dust properties of far-infrared cavities namely FIC16-37 and FIC12-58 nearby carbon-rich AGB stars, AGB15-38 and AGB12-57, are studied. For that, the dust color temperature, dust mass, Planck function, and visual extinction of the region of interest are evaluated. Moreover, the variation of Planck function along compression and extension of the cavities are also studied, which is crucial for understanding the thermal stability in the ISM.

\section{Far Infrared Cavity}

This paper presents a systematic search and study of two new far-infrared cavities around the 1168 Carbonrich (C-rich) AGB stars provided in the catalogue of Suh \& Kwon [11]. Far infrared cavities, namely FIC16-37 (R.A. (J2000): $16^{h} 33^{m} 57.25^{s}$ \& Dec. (J2000): $-37^{d} 47^{m} 04.3^{s}$ ), and FIC12-58 (R.A. (J2000): $12^{h} 52^{m} 50.08^{s}$ \& Dec. (J2000): $-58^{d} 08^{m} 55.02^{s}$ ) nearby AGB stars, AGB15-38 (R.A. (J2000): $15^{h} 37^{m} 40.74^{s}$ \& Dec. (J2000): $-38^{d} 20^{m} 24.6^{s}$ ), and AGB12-57 (R.A (J2000): $12^{h} 56^{m} 38.50^{s} \&$ Dec. (J2000): $-57^{d} 54^{m} 34.70^{s}$ ) respectively, at $60 \mu \mathrm{m}$ and $100 \mu \mathrm{m}$ IRAS map were found. The selection criteria were as follows: (1) cavity should be isolated from any other cavities or loops, (2) major diameter should be $>0.5^{\circ}$, (3) the region should lie within $-10^{\circ}<\mathrm{b}<10^{\circ}$ in Galactic planes and (4) no diffuse optical emission (surrounding brightness limit $\sim 5 \mathrm{mag} / \mathrm{arcsec}^{2}$ with resolution $2 \operatorname{arcsec}$, and size $1.7 \mathrm{sec} / \mathrm{pixel}$ ) [22]. This research work is constrained to the study of cavities within $-10^{\circ}<\mathrm{b}<10^{\circ}$ Galactic planes because it is believed that there is a possibility of high-pressure events within this range [22]. The FITS images $(100 \mu \mathrm{m} \& 60 \mu \mathrm{m})$ were downloaded from SkyView Virtual Observatory and calculated the flux density at each pixel of the cavity using software ALADIN 2.5. Fig. 1(a) and 3(a) show the JPG image of cavities $\left(1.0^{\circ} \times 1.0^{\circ}\right)$ FIC16-37 and FIC12-58 having an extension and compression $\sim 4.79 \mathrm{pc} \& \sim 3.06 \mathrm{pc}$, and $\sim 22.5$ pc \& $\sim 14.84$ pc respectively. The distance to the FIC16-37 and FIC12-58 is $\sim 390.76$ pc and $\sim 1342$ pc respectively, which was estimated using Gaia DR2 (parallax value) (https://www.cosmos.esa.int/web/gaia/dr2).

\section{Methodology}

This paper includes the study of dust properties of the dusty environment of cavities around carbonrich AGB stars. The detail methods for calculating dust color temperature, Planck function, dust mass, visual extinction, and size of the structure are explained in details below.

\subsection{Dust color temperature}

The dust color temperature was calculated using the method proposed by Wood et al. [13], and later improved by Dupac et al. [23], and Schnee et al. [24]. The dust color temperature of each pixels of FITS image was calculated using $60 \mu \mathrm{m}$ and $100 \mu \mathrm{m}$ IRAS flux densities. The expression for dust color temperature $T_{d}$ is:

$$
T_{d}=\frac{-96}{\ln \left\{R \times 0.6^{3+\beta}\right\}} \quad \text { where, } R=\frac{F(60 \mu m)}{F(100 \mu m)}
$$


Here, $\beta$ is the spectral emissivity index which depends upon the dust grain properties like compression, size, and compactness. $\beta=0$ for the black body, $\beta \sim 1$ for amorphous layer-lattice, and $\beta=2$ for crystalline dielectrics which were used in our calculation. $F(60)$ and $F(100)$ represent flux densities in $60 \mu \mathrm{m}$ and $100 \mu \mathrm{m}$ respectively.

\subsection{Planck function}

The expression for Planck function is given by Beichman et al. [25]. It depends on wavelength, and hence temperature. Its value is high for longer wavelength and small for shorter wavelength and is calculated as:

$$
B(\nu, T)=\frac{2 h c}{\lambda^{3}}\left(\frac{1}{e^{\frac{h c}{\lambda K T}-1}}\right)
$$

This value is used to calculate the dust mass of the region under study. Moreover, the range of $B(\nu, T)$ for fixed temperature goes narrower if the wavelength of images increases.

\subsection{Dust mass}

The dust mass for each pixel is calculated using the expression given by Hildebrand [26]. There is a use of flux density $F(\nu)$, Planck function $B(\nu, T)$, and distance to the structure $(D)$ for its calculation and is given as:

$$
M_{d}=\frac{4 a \rho}{3 Q(\nu)} \cdot \frac{F(\nu) D^{2}}{B(\nu, T)}
$$

where, values of weighted grain size $(a)=0.1 \mu \mathrm{m}$, grain density $(\rho)=3000 \mathrm{kgm}^{-3}$, grain emissivity $(\mathrm{Q}(\nu))=$ 0.0010 for $100 \mu \mathrm{m}[27]$ are used. Thus, above expression reduces as:

$$
M_{d}=0.4\left[\frac{F(\nu) D^{2}}{B(\nu, T)}\right]
$$

\subsection{Visual Extinction}

This paper evaluates the visual extinction based upon the empirical formula given by Wood et al. [13]. According to them, visual extinction is given as:

$$
A_{\nu}(\mathrm{mag})=15.078\left[1-e^{-\tau_{100} / 641.3}\right] \quad \text { where, } \tau_{100}=\frac{F(100 \mu m)}{B\left(\nu, T_{d}\right)}
$$

Where $\tau_{100}, F(100 \mu \mathrm{m})$, and $B\left(\nu, T_{d}\right)$ are the optical depth at $100 \mu \mathrm{m}$ wavelength, the flux at $100 \mu \mathrm{m}$, and Planck function at $100 \mu \mathrm{m}$, respectively.

\subsection{Size of the structure}

The size of the cavity structure is calculated following the method, as indicated in Gautam \& Aryal [28]. The expression for the size of the structure is given by,

$$
R=D \times \theta
$$

where, $D$ is the distance to the structure, and $\theta$ is the pixel size in radian. 


\section{Result and discussion}

We describe the physical properties of two far-infrared cavities, FIC16-37 (R.A. (J2000): $16^{h} 33^{m} 57.25^{s} \&$ Dec. (J2000): $-37^{d} 47^{m} 04.3^{s}$ ) and FIC12-58 (R.A. (J2000): $12^{h} 52^{m} 50.08^{s} \&$ Dec. (J2000): $-58^{d} 08^{m} 55.02^{s}$ ), and compare with the previously published findings.

\subsection{FIC16-37}

Fig. 1(a) shows the JPG image of cavity FIC16-37 at 100 micron IRAS map where the central position of the cavity is indicated by symbol ' + ' . Figs. 1(b), 1(d) and 1(f) represent the contour map of flux density (100 micron), dust color temperature, and dust mass whereas Figs. 1(c) and 1(h) represent the linear fit between $F(100) \& F(60)$, and $A_{v} \& T_{d}$ respectively of the cavity structure. The flux densities at 60 and 100 microns were obtained using ALADIN 2.5 software. The background flux contributed by the nearby stars and IRAS sources were subtracted. For this, the SIMBAD (http://simbad.u-strasbg.fr/) database was used. The flux regions were separated into different clusters. The minimum flux-region was found to lie at the central region of the cavity. The slope of the best fit line between flux densities, as shown in Fig. 1(c) was used to set the error bars [21]. The dust color temperature $\left(T_{d}\right)$ of each pixel was evaluated using Eq. (1) and found in the range (23.95 \pm 0.25$)$ $\mathrm{K}$ to $(23.44 \pm 0.27) \mathrm{K}$ with offset about $0.5 \mathrm{~K}$. This narrower temperature range clearly indicated that there is the symmetric outflow or symmetric distribution of density and temperature, suggesting that the particles are independently vibrating and it may be in thermally pulsating phase [29]. The majority of minimum flux regions corresponded to minimum temperature region, but some minimum regions at the northern part of the cavity had a larger temperature as shown in Fig. 1(d), which is possibly due to the effect of nearby sources (e.g. AGB wind). The total mass of the cavity structure was found to be $1.06 \times 10^{29} \mathrm{~kg}\left(0.053 \mathrm{M}_{\odot}\right)$ with average mass $1.89 \times 10^{26} \mathrm{~kg}$. From the contour map of dust color temperature and dust mass, the cosmological principle is followed as the majority of the minimum temperature regions correspond to the denser mass regions, indicating a homogeneous and isotropic distribution of dust [30]. The Gaussian fit of temperature and mass showed Gaussianlike behaviour with Gaussian centres $23.70 \mathrm{~K}$ and $1.86 \times 10^{26} \mathrm{~kg}$, respectively. This indicated the higher possiblity of dust particles being in local thermodynamical equilibrium. Fig. 1(h) shows the scatter plot of $A_{v}$ with respect to $T_{d}$. The majority of the data points were concentrated towards the line of fit, indicating a possible linear relationship between $A_{v}$ and $T_{d}$ with negative correlation $(\mathrm{R})=-0.47$. Thus, the product of $T_{d}$ and $A_{v}$ was found to be less than 1, consistent with the previous findings [21, 28, 29]. Moreover, this low value of correlation gives high emphasis on the possible role of AGB wind. Clearly value of $A_{v}$ must be greater in denser region, which implies less value of temperature in the corresponding region. But, due to possible interaction with AGB wind the minimum temperature and maximum mass regions don't concide exactly, giving rise to less value of correlation. The major and minor diameters of the cavity were calculated using the Eq. (6), which was found to be $\sim 4.79$ $\mathrm{pc}$ and $\sim 3.06 \mathrm{pc}$, respectively. 
Fig. 2(a) and Fig. 2(b) show the variation of Planck functions along major and minor diameters passing through the minimum flux region of the cavity. The error bars represent the standard error (SE) of the deviation. The Planck function was found to be non-uniform along both of the diameters and followed sinusoidal distribution. This result is consistent with the findings of Gautam \& Aryal [1] and Gautam et al. [17]. This suggests that the particles are not in thermal equilibrium along both diameters and oscillating to acquire dynamical equilibrium. But, Jha et al. [20] obtained uniform distribution of Planck function along major and minor diameters in case of cavities nearby pulsars which emits radiation. This suggests that there might be the role of other nearby sources (e.g. AGB wind) over the FIC16-37 cavity region.
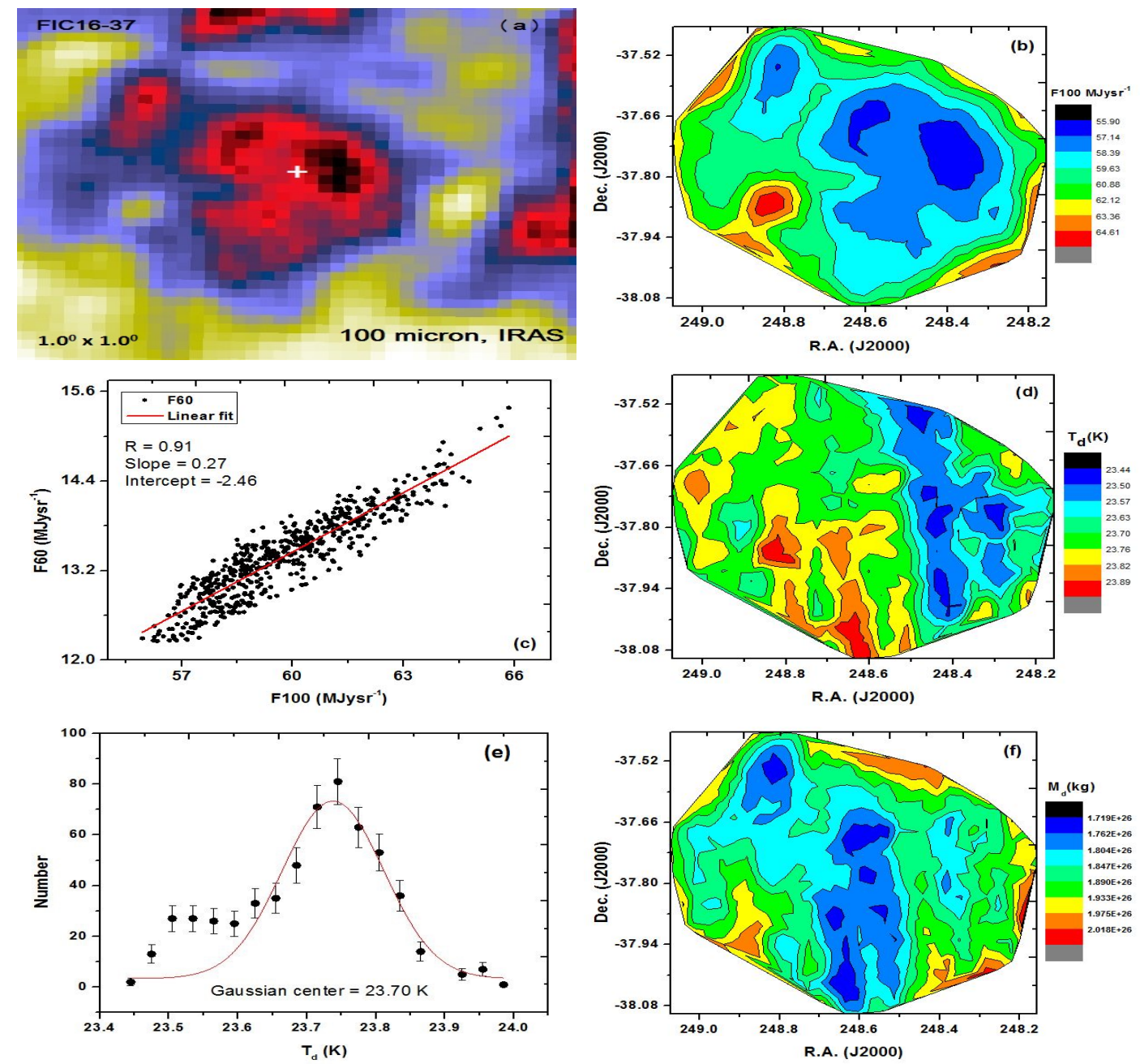

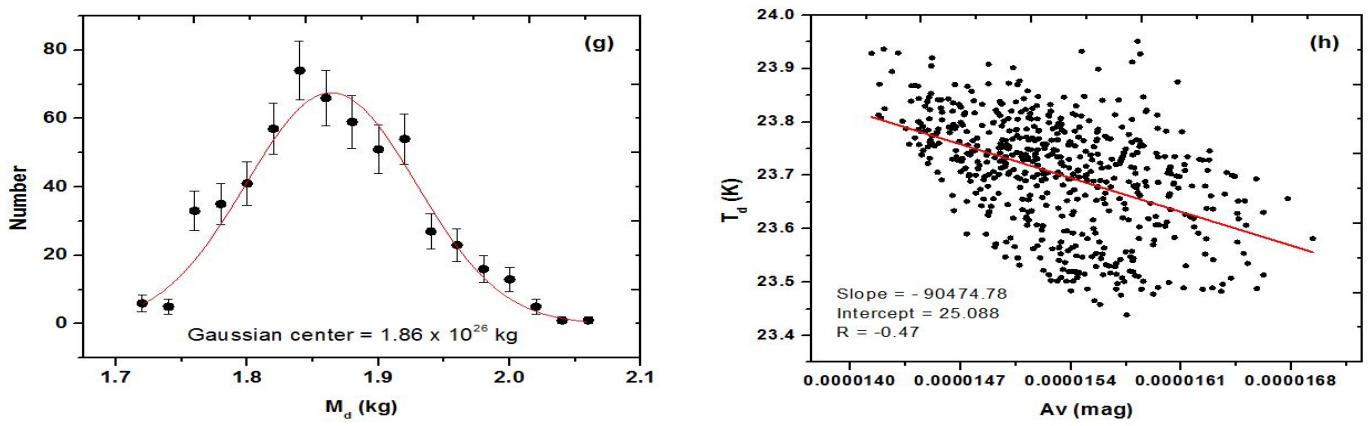

Figure 1. (a) JPEG image (b) Contour map of the flux density at $100 \mu \mathrm{m}$ IRAS map (c) The linear fit of 100 $\mu \mathrm{m}$ vs $60 \mu \mathrm{m}$ flux density (d) Contour map of dust color temperature (e) Gaussian fit of dust color temperature (f) Contour map of dust mass (g) Gaussian fit of dust mass (h) Linear fit of scatter plot between visual extinction $\left(A_{v}\right)$ and dust color temperature $\left(T_{d}\right)$, of the far-infrared cavity FIC16-37 centred at R.A (J2000): $16^{h} 33^{m} 57.25^{s}$ and Dec. (J2000): $-37^{d} 47^{m} 04.3^{s}$ nearby AGB12-38 at 100 $\mu \mathrm{m}$ IRAS map.
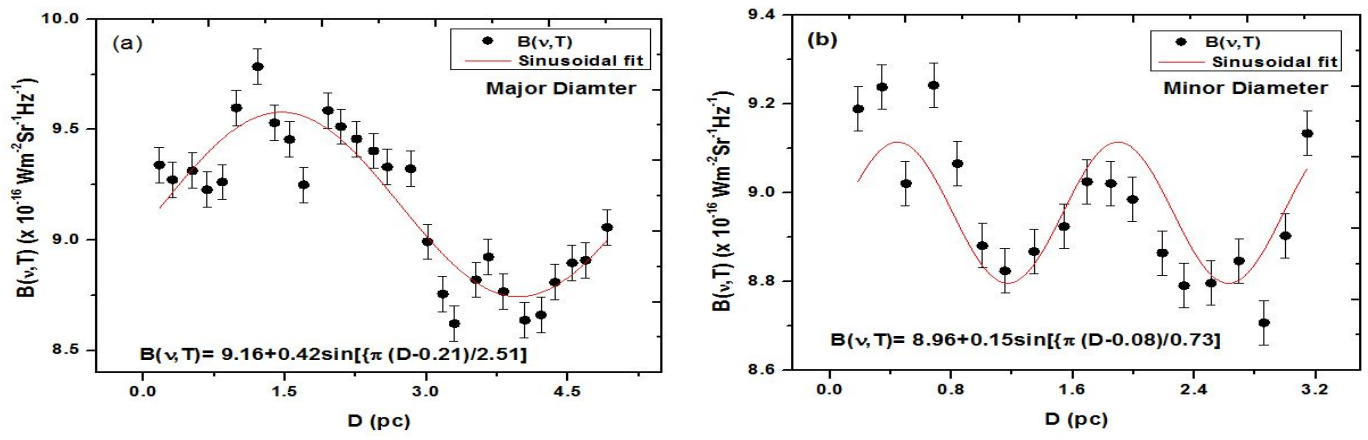

Figure 2. Variation of Planck function $(B(\nu, T))$ of the cavity FIC16-37 with the distance along (a) major diameter and (b) minor diameter.

\subsection{FIC12-58}

The symbol ' +' in Fig. 3(a) shows the central position of the cavity FIC12-58 at 100 microns IRAS map. Figs. 3(b), 3(d) and 3(f) show the contour map of flux density (100 microns), dust color temperature and dust mass of the cavity structure, respectively. Figs. 3(c) and 3(h) show the linear fit between $F(100) \& F(60)$ and $A_{v} \& T_{d}$ respectively. The flux densities at 60 and 100 microns were obtained using the software ALADIN 2.5. The possible background flux for this cavity was also subtracted. The minimum flux-region for this cavity was also found in the central region, as shown in Fig. 3(b). Similar to the previous cavity, the slope of the best fit line between flux densities as shown in Fig. 3(c) was used to set the error bars. The dust color temperature $\left(T_{d}\right)$ was found to be in the range $(24.88 \pm 0.27) \mathrm{K}$ to $(23.63 \pm 0.98) \mathrm{K}$ with offset about $1 \mathrm{~K}$. The low offset in this cavity also suggests the higher possibility of symmetric outflow or symmetric distribution of density and temperature. This indicates that the particles are independently vibrating and it may be in a thermally pulsating phase similar to cavity FIC12-58. The minimum flux region of this cavity corresponds to moderate temperature region which indicates the possible effects of nearby sources (e.g AGB wind). The total mass of this cavity was found to be 
$1.56 \times 10^{30} \mathrm{~kg}\left(0.78 \mathrm{M}_{\odot}\right)$, with an average mass of $1.63 \times 10^{27} \mathrm{~kg}$. The contour map of dust color temperature and dust mass of this cavity shows that the dust distribution also follows the cosmological principle as maximum temperature region corresponds to a less denser mass region and vice-versa. This also indicates the homogeneous and isotropic distribution of dust around the cavity. The Gaussian fit of temperature and mass of this cavity also show the Gaussian-like behaviour similar to FIC16-37 with Gaussian centre $24.21 \mathrm{~K}$ and $1.61 \times 10^{27} \mathrm{~kg}$, indicating the higher possiblity of dust particles being in local thermodynamic equlibrium as in the cavity FIC16-37. Fig. $3(\mathrm{~h})$ shows the scatter plot of $A_{v}$ with respect to $T_{d}$. The majority of the data points were concentrated towards the line of fit indicating a possible linear relationship between $A_{v}$ and $T_{d}$ with negative correlation $(\mathrm{R})=-0.43$ satisfying $A_{v} \times T_{d}<1$. This also supports the previous literatures. The major and minor diameters of this cavity were found to be $\sim 22.54 \mathrm{pc}$ and $\sim 14.84 \mathrm{pc}$, respectively.

Fig. 4(a) and Fig. 4(b) show the variation of Planck functions along major and minor diameters passing through minimum flux region of the cavity FIC12-58. Unlike cavity FIC16-37, the line of best fits was different. Along major diameter, the line of best fit was found to be linear; however, along the minor diameter was found to be sinusoidal. The nature of vibration of particles is different along the diameters indicating the role of nearby sources (possibly AGB wind) wind different than their role in case of cavity FIC12-58. The error bars represent the standard error (SE) of the deviation. The result for the major diameter is in good agreement with the result of Gautam \& Aryal [28] and for the minor diameter was found to be consistent with the result of Gautam \& Aryal [1]. These variations of Planck functions suggest that the particles in this cavity are also not in thermal equilibrium along both diameters.
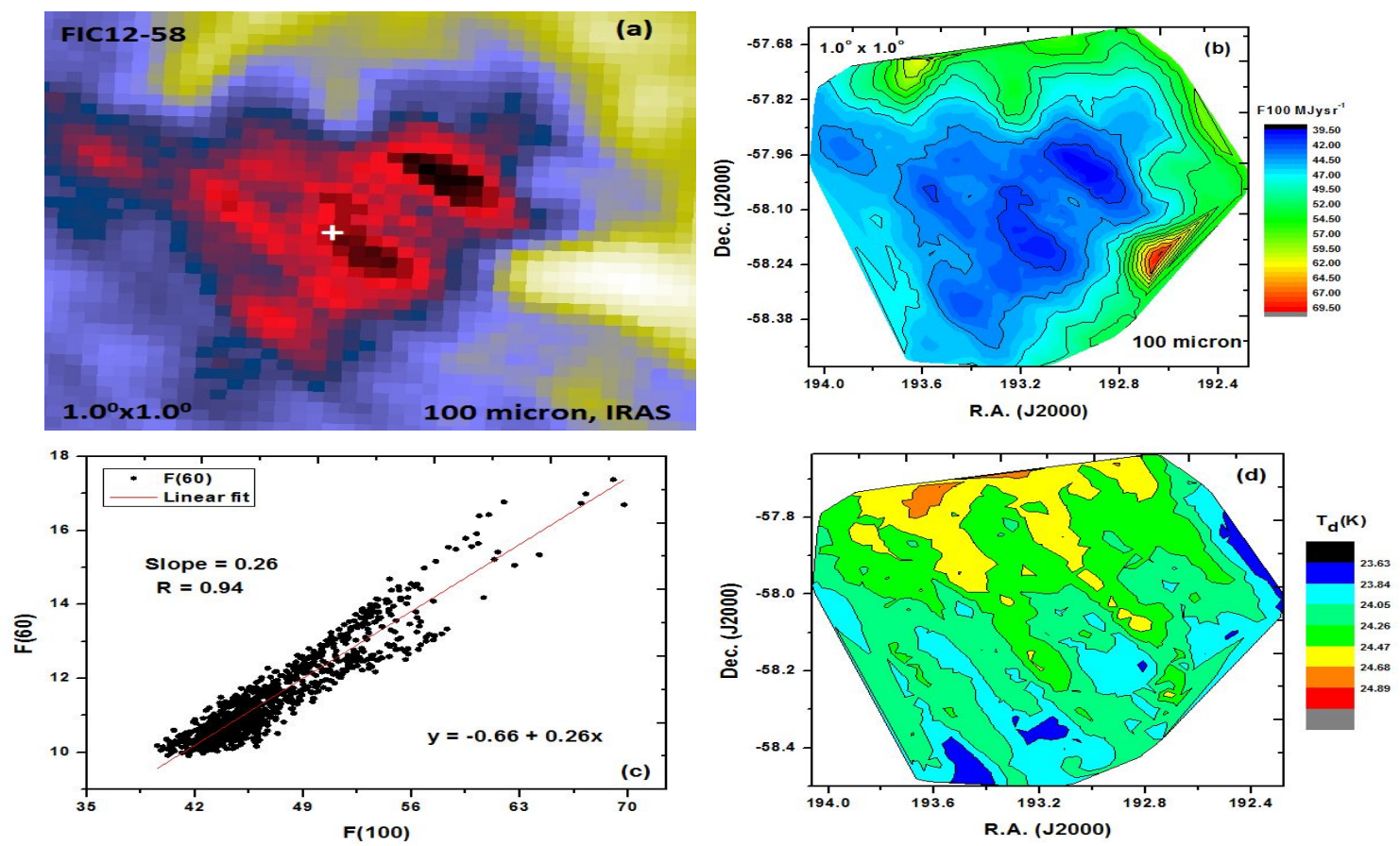

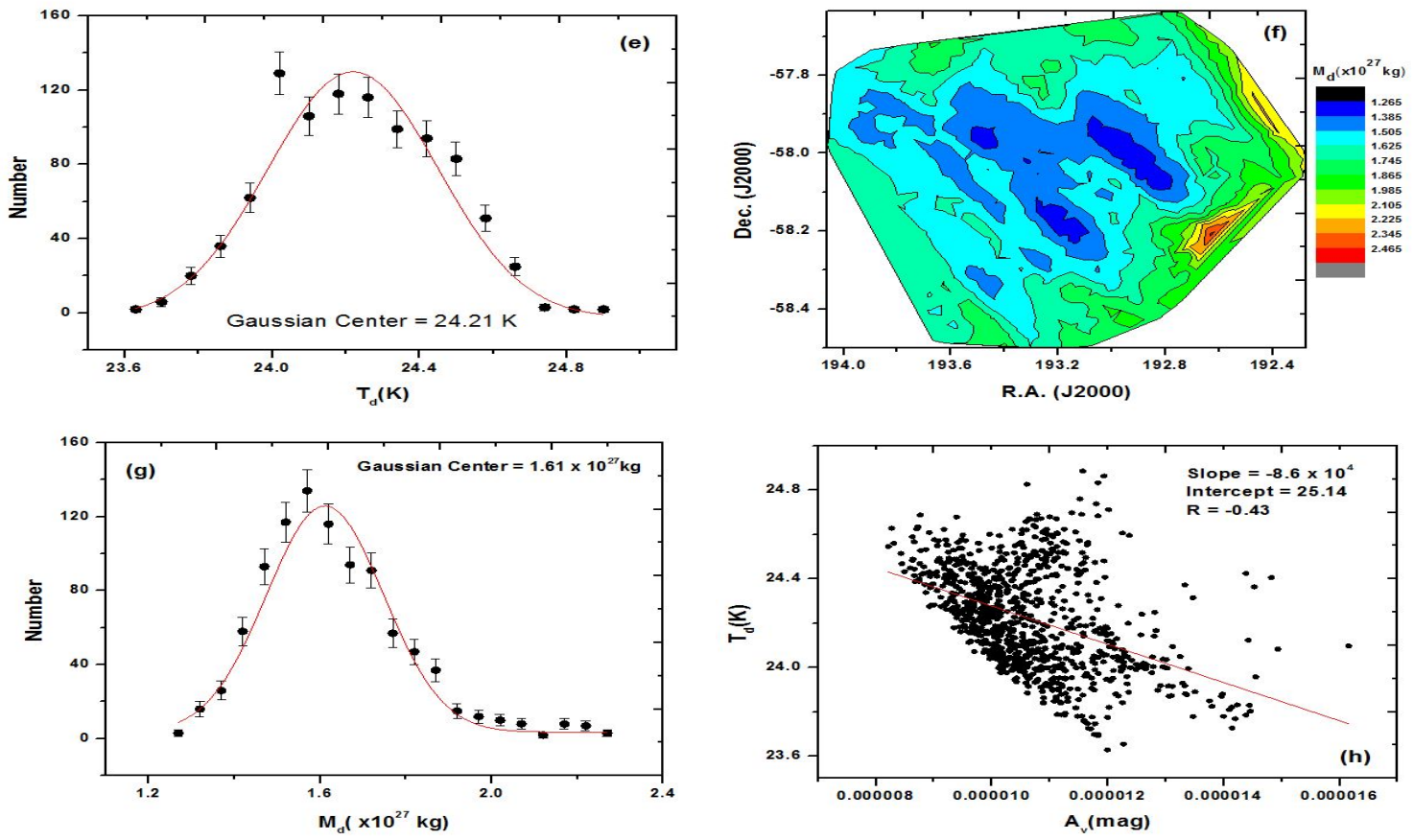

Figure 3. (a) JPEG image (b) Contour map of the flux density at $100 \mu \mathrm{m}$ IRAS map (c) The linear fit of 100 $\mu \mathrm{m}$ vs $60 \mu \mathrm{m}$ flux density (d) Contour map of dust color temperature (e) Gaussian fit of dust color temperature (f) Contour map of dust mass (g) Gaussian fit of dust mass (h) Linear fit of scatter plot between visual extinction $\left(A_{v}\right)$ and dust color temperature $\left(T_{d}\right)$, of the far-infrared cavity FIC12-58 centred at R.A (J2000): $12^{h} 52^{m} 50.08^{s}$ and Dec. (J2000): $-58^{d} 08^{m} 55.02^{s}$ nearby AGB12-38 at 100 $\mu \mathrm{m}$ IRAS map.
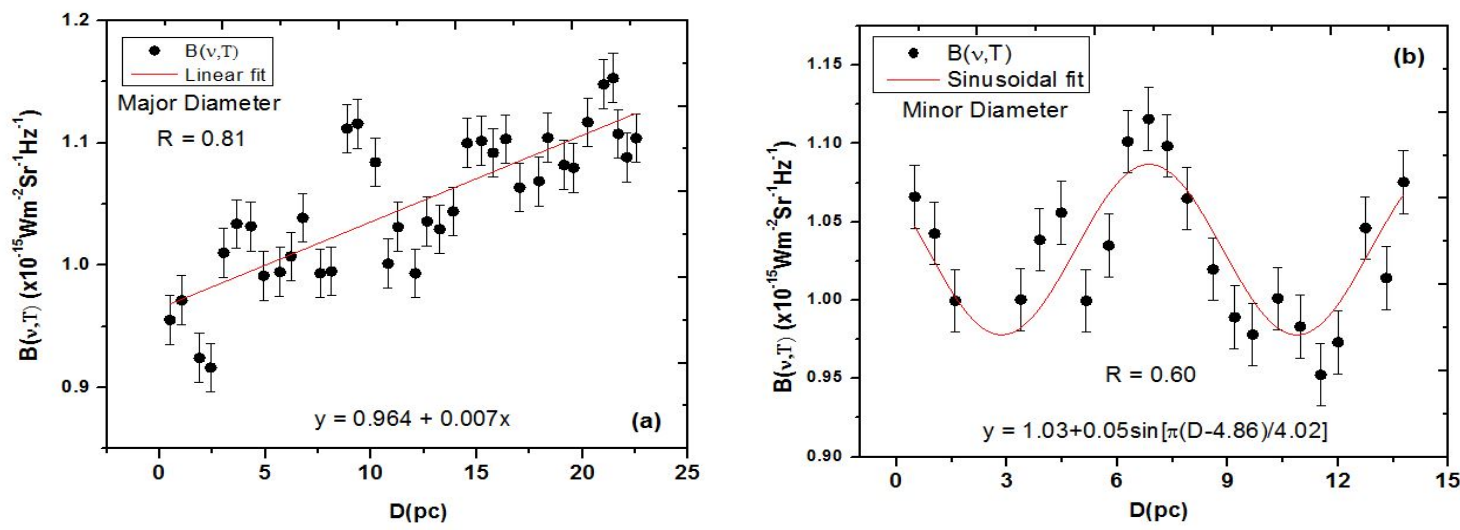

Figure 4. Variation of Planck function $(B(\nu, T))$ of the cavity FIC12-58 with the distance along (a) major diameter and (b) minor diameter.

\section{Conclusion}

We studied the physical properties such as dust color temperature, dust mass, visual extinction, Planck function, and size of the structure of two new far-infrared cavities, FIC16-37 (R.A. (J2000): $16^{h} 33^{m} 57.25^{s} \&$ 
Dec. (J2000): $-37^{d} 47^{m} 04.3^{s}$ ), and FIC12-58 (R.A. (J2000): $12^{h} 52^{m} 50.08^{s} \&$ Dec. (J2000): $-58^{d} 08^{m} 55.02^{s}$ ) nearby Carbon-rich AGB stars using 60 and 100 micron IRAS maps. Conclusions of the results are as follows:

- The major and minor diameters of the far-infrared cavities, namely FIC16-37 and FIC12-58, were found to be $\sim 4.79$ pc $\& \sim 3.06$ pc, and $\sim 22.54$ pc $\& \sim 14.84$ pc respectively.

- The value of dust color temperature was found to lie in the range of $23.95 \pm 0.25 \mathrm{~K}$ to $23.44 \pm 0.27 \mathrm{~K}$ with an offset of about $0.5 \mathrm{~K}$ for the cavity FIC16-37 and $24.88 \pm 0.27 \mathrm{~K}$ to $23.63 \pm 0.98 \mathrm{~K}$ with an offset of about $1 \mathrm{~K}$ for the cavity FIC12-58. This small value of offset in both of the cavities indicated the symmetric outflow or symmetric distribution of density and temperature.

- The total mass of the cavities FIC16-37 and FIC12-58 were found to be $0.053 \mathrm{M}_{\odot}$ and $0.78 \mathrm{M}_{\odot}$, respectively. The contour map of dust color temperature and dust mass showed that the minimum temperature region corresponds to a dense mass region and vice-versa, following the cosmological principle. It further suggests that the distribution of masses were homogeneous and isotropic for both cavities.

- A fluctuation in the distribution of Planck function along extension and compression indicated the role of nearby sources (possibly AGB wind) to both of the cavities. These fluctuations were random and indicated deviation of dust from local thermodynamic equilibrium. The variation of Planck function along major and minor diameters of FIC16-37 was sinusoidal. However, in the FIC12-58, the variation of Planck function distribution along the major diameter was linear with sinusoidal distribution in minor diameter. This resulted consistency in the nature of oscillation of dust particles in FIC16-37 cavity along the diameters but inconsistency in case of FIC12-58. This suggested the difference in nature of the interaction of cavities with AGB wind.

- However, we did not obtain a strong negative correlation between $\mathrm{A}_{v}$ and $\mathrm{T}_{d}$, which is possibly due to interaction of AGB wind with the intestellar medium. We found the concentration of the majority of the data points to a particular region indicating $A_{v} \times T_{d}<1$ for both cavities.

This study serves as an essential reference to carry out further studies about the shaping mechanism of Interstellar Medium. The possible interaction of these cavities with the AGB stars or nearby sources need to be studied. In the future, we will investigate the amount of expelled mass from the central region in order to estimate the energy required for the structure formation.

\section{References}

[1] Gautam A, Aryal B. A study of four low-latitude $\left(|l|<10^{\circ}\right)$ far-infrared cavities. Journal of Astrophysics and Astronomy. 2019;40(2):1-10. 
[2] Iben Jr I. Stellar Evolution. VI. Evolution from the Main Sequence to the Red-Giant Branch for Stars of Mass 1 M_ sun\}, 1.25 M_ sun\}, and 1.5 M_ sun\}. The Astrophysical Journal. 1967;147:624.

[3] Bertulani C, Kajino T. Frontiers in nuclear astrophysics. Progress in Particle and Nuclear Physics. 2016;89:56100.

[4] Vassiliadis E, Wood P. Evolution of low-and intermediate-mass stars to the end of the asymptotic giant branch with mass loss. The Astrophysical Journal. 1993;413:641-657.

[5] Forestini M, Goriely S, Jorissen A, Arnould M. Fluorine production in thermal pulses on the asymptotic giant branch. Astronomy and Astrophysics. 1992;261:157-163.

[6] Aryal B, Weinberger R. Dust Structure Around White Dwarf WD 1003-44 in 60 \& $100 \mu$ m Iras Survey. Himalayan Physics. 2011;2:5-10.

[7] Marigo P, Girardi L, Bressan A, Groenewegen MA, Silva L, Granato GL. Evolution of asymptotic giant branch stars-II. Optical to far-infrared isochrones with improved tp-agb models. Astronomy \& Astrophysics. 2008;482(3):883-905.

[8] Gallino R, Arlandini C, Busso M, Lugaro M, Travaglio C, Straniero O, et al. Evolution and nucleosynthesis in low-mass asymptotic giant branch stars. II. Neutron capture and the s-process. The Astrophysical Journal. $1998 ; 497(1): 388$.

[9] Mowlavi N. On the third dredge-up phenomenon in asymptotic giant branch stars. arXiv preprint astroph/9903473. 1999;.

[10] Rees MJ. Black hole models for active galactic nuclei. Annual review of astronomy and astrophysics. $1984 ; 22(1): 471-506$.

[11] Suh KW, Kwon YJ. A Catalog of AGB Stars in IRAS psc. Journal of Korean Astronomical Society. 2009;42:81-91.

[12] Suh KW, Kwon YJ. Infrared two-colour diagrams for AGB stars using AKARI, MSX, IRAS and near-infrared data. Monthly Notices of the Royal Astronomical Society. 2011;417(4):3047-3060.

[13] Wood DO, Myers PC, Daugherty DA. IRAS images of nearby dark clouds. The Astrophysical Journal Supplement Series. 1994;95:457-501.

[14] Odenwald SF, Rickard LJ. Hydrodynamical processes in the Draco molecular cloud. The Astrophysical Journal. 1987;318:702-711.

[15] Odenwald SF. Comet-like clouds at far-infrared and optical wavelengths-Mach cones and hydrodynamics? The Astrophysical Journal. 1988;325:320-341.

[16] Weinberger R, Armsdorfer B. A pair of gigantic bipolar dust jets close to the solar system. Astronomy \& Astrophysics. 2004;416(3):L27-L30.

[17] Gautam SP, Silwal A, Jha AK. Dust Color Temperature and Planck Function Distribution of a Far Infrared Planetary Nebula at 90 and $140 \mu \mathrm{m}$ AKARI Map. International Astronomy and Astrophysics Research Journal. 2020;2(2):1-8. 
[18] Aryal B, Rajbahak C, Weinberger R. A giant dusty bipolar structure around the planetary nebula NGC 1514. Monthly Notices of the Royal Astronomical Society. 2010;402(2):1307-1312.

[19] Aryal B, Rajbahak C, Weinberger R. Planetary nebulae NGC 6826 and NGC 2899: early aspherical mass loss? Astrophysics and Space Science. 2009;323(4):323-327.

[20] Jha A, Aryal B, Weinberger R. A study of dust color temperature and dust mass distributions of four far infrared loops. Revista mexicana de astronomía y astrofísica. 2017;53(2).

[21] Jha A, Aryal B. Dust color temperature distribution of two FIR cavities at IRIS and AKARI maps. Journal of Astrophysics and Astronomy. 2018;39(2):24.

[22] Lasker BM, Sturch CR, McLean BJ, Russell JL, Jenkner H, Shara MM. The Guide Star Catalog. IAstronomical foundations and image processing. The Astronomical Journal. 1990;99:2019-2058.

[23] Dupac X, Bernard JP, Boudet N, Giard M, Lamarre JM, Mény C, et al. Inverse temperature dependence of the dust submillimeter spectral index. Astronomy \& Astrophysics. 2003;404(1):L11-L15.

[24] Schnee SL, Ridge NA, Goodman AA, Li JG. A complete look at the use of IRAS emission maps to estimate extinction and dust temperature. The Astrophysical Journal. 2005;634(1):442.

[25] Beichman C, Wilson R, Langer W, Goldsmith P. Infrared limb brightening in the Barnard 5 cloud. The Astrophysical Journal. 1988;332:L81-L85.

[26] Hildebrand RH. The determination of cloud masses and dust characteristics from submillimetre thermal emission. 1983;

[27] Young K, Phillips T, Knapp G. Circumstellar shells resolved in IRAS survey data. II-Analysis. The Astrophysical Journal. 1993;409:725-738.

[28] Gautam A, Aryal B. Study of Two Far Infrared Cavities Nearby Asymptotic Giant Branch Stars Under Infrared Astronomical Satellite Maps. Journal of Institute of Science and Technology. 2019;24(2):76-84.

[29] Gautam A, Aryal B. Study of dust color temperature and visual extinction distribution of a far infrared cavity at 60 and $100 \mu \mathrm{m}$ IRAS map around the AGB star at galactic latitude 8.6 ${ }^{\circ}$. BIBECHANA. 2020;17:42-49.

[30] Sapkota B, Aryal B. A Study of Far Infrared Cavity At-3.6 ${ }^{\circ}$ Galactic Latitude. Journal of Nepal Physical Society. 2019;5(1):54-58. 